\title{
Performance Analysis of MIMO-OFDM System with Relaying Techniques in Rayleigh Fading Channel
}

\author{
Shivi Tikko*1, Shweta Pandit*², Pradeep Kumar ${ }^{\# 3}$ \\ * Department of Electronics and Communication Engineering \\ Jaypee University of Information Technology, Waknaghat-173234, India \\ 1shivitikoo@gmail.com \\ ${ }^{2}$ shweta.pandit@juit.ac.in \\ ${ }^{*}$ Discipline of Electrical, Electronic and Computer Engineering \\ University of KwaZulu-Natal, Howard College Campus, King George V Avenue, Durban-4041, South Africa \\ ${ }^{3}$ kumarp@ukzn.ac.za
}

\begin{abstract}
A relay network is a wide class of network configuration which is often used in wireless networks, where the source node and destination node cannot communicate directly and are interlinked with the help of some nodes. The different methods by which the relay networks are implemented in the system are known as relaying techniques. In this paper, we have presented the spectral efficiency and BER performance analysis of the MIMO-OFDM wireless system with different relaying techniques in Rayleigh fading channel. The simulated results for different modulation schemes are presented. The obtained results have shown that the hybrid relaying technique provides better spectral efficiency and bit error rate (BER) performance as compared to the other relaying techniques.
\end{abstract}

Keyword-MIMO-OFDM, relaying techniques, decode and forward, amplify and forward, hybrid decode and amplify forward, Rayleigh fading, spectral efficiency, BER.

\section{INTRODUCTION}

The use of diverse number of antennas at the transmitter and receiver side in wireless system is known as Multiple Input and Multiple Output technology or MIMO technology that has achieved popularity over the past years because of its powerful performance enhancing capabilities over single input single output or SISO antenna system. MIMO antenna technology exploits the spatial domain property of fading channels to bring noteworthy performance improvements to wireless communication systems. Typical MIMO systems require both the transmitter and receiver of a communication link to be equipped with multiple antennas and have potential for increased capacity [1-3]. In addition, MIMO system improves SNR, range and coverage area of a wireless network [4]. It also improves the quality and reliability of reception and is resistant to noise [5]. Further, in OFDM (Orthogonal Frequency Division Multiplexing) system, large amount of closely spaced orthogonal subcarrier signals carry data on various data channels or data streams. Each sub-carrier signal is modulated by suitable modulation scheme like QAM, PSK or FSK at a low symbol rate, thus maintaining the total data rate same as that of standard single carrier modulation scheme for the same bandwidth. OFDM is advanced form of FDM (Frequency Division Multiplexing) where all subcarrier data signals are orthogonal to one another. The signals whose integral of the products for their common (fundamental) period is zero are known as orthogonal signals [6]. The main advantage of OFDM system is that it makes competent use of the spectrum by enabling the overlapping of signals and divides the broadband frequency selective channel into various narrowband flat fading channels. Hence, as compared to single carrier systems, OFDM is more impervious to frequency selective fading.

All wireless linkage are mainly affected by three typical parameters which are speed, range and reliability. These characteristics of wireless system are tied to each other by strict regulations. Speed could be enhanced only by neglecting the range and reliability of the system. Range could be widen at the loss of reliability and speed. And at last, reliability could be reinforced by deflating range and speed. Only MIMO-OFDM gives everything in one set by offering optimal values of speed, range and reliability simultaneously. In OFDM, an individual channel within a spectrum band can be segmented into large number of small sub carrier signals that can transmit information simultaneously without any kind of interference. Since in MIMO technology many smaller antennas are linked up to work as one, so they can transmit and receive these multiple OFDM sub signals. This allows the substantial increment of bandwidth for each user as required. Thus, combining these two technologies results in a wireless system that has characteristics like good spectral coverage, more reliable transmission in highly perverse surrounding, with high data rates [7, 8]. Since MIMO serves the spatial diversity whereas OFDM uses multiplexing technique like frequency division duplex (FDD) or time division duplex (TDD); therefore in the spatial domain, MIMO provides greater capacity. Further in the time domain, the 
equalization process is also simplified by OFDM system by eliminating ISI. Therefore, channel capacities could be enhanced and realized with robustness with the merger of MIMO system with OFDM system.

In addition to this, a relay network is a boundless classification of network topology that is used in wireless networks where relay nodes establish a connection between source and destination [9]. These relay nodes are required in a network where the source and destination cannot convey information directly as the distance between them is greater than their transmission range. Relayed transmission improves the quality of wireless communications and some of the advantages of relay network in comparison with direct link communication are: good scalability, increased connectivity, extended transmission range, increased throughput, and improved reliability. Different methods known as relaying techniques by which the relay networks are implemented are described below [10]:

i. Amplify and Forward (AF) Relay Technique: In this technique, relay nodes straightforwardly amplify the signal received from the source node which includes both the desired signal and undesired signal which is added noise. Further the relay node transmits it to the destination node. Therefore, AF suffers with the noise amplification problem. This degrades the signal quality particularly at low values of SNR. However, it is the simplest relay technique to implement [11].

ii. Decode and Forward (DF) Relay Technique: In this technique, the relay node eliminates the noise by decoding the received signal, then regenerates and re-encodes the signal and transmits it further to the destination. However, DF suffers with the error propagation problem which occurs if the relay incorrectly decodes the information and forwards this incorrect data to the destination node [12].

iii. Hybrid Decode Amplify and Forward (HDAF) Relay Technique: This relaying technique collaborates the two DF and AF relaying techniques. The hybrid relay node makes transition between DF and AF depending on the channel conditions. Further, HDAF protocol gives less erroneous output as compared to other relaying techniques [13].

iv. Demodulate and forward Relay Technique: Demodulate-and-forward relaying technique is basically an enhanced version of DF that reduces the amount of power consumed by the receiver or the destination node due to channel decoding at the relay. This also minimizes the overall delay at the destination [14].

v. Incremental Relay Technique: Incremental relaying is basically dependent on the feedback process. In this relay protocol, the relay node performs re-transmission when destination node does not receive the data correctly. This scheme enhances the performance of AF, DF, HDAF relaying by exploiting the feedback from the destination node and relay node whenever necessary [15].

vi. Best-Relay Selection Relay Technique: In such a relay protocol, the "best” relay link among all relay nodes is selected to transmit data to the destination node. The best relay that is selected achieves high SNR at the destination node. This also maintains a full diversity order [16].

In the context of above discussion, MIMO-OFDM relay communication systems have received considerable research interest in the past years by improving the link reliability, capacity and extend the network coverage. Some literature which has used relaying techniques for improving the performance of communication system has been detailed further. In [17], the strengthening of security of multi-user relay network with multiple number of AF relay links in the presence of eavesdroppers has been discussed. The criteria of selecting the best relay node and destination node pair is evaluated by increasing the SNR of user as compared to the eavesdropper. Another proposed technique to achieve full diversity order with whichever number of selected antennas among all available antennas in the relay network with DF relay nodes has been studied in [18]. Authors discussed about new transmit antenna selection called source antenna selection (STAS) which is based on CSI (Channel State Information) as well as on the transmission technique of MIMO-DF network. Further in [19], HDAF and incremental relaying is collaborated to form a new relay protocol known as IHDAF (incremental-HDAF) where the relay node chooses between AF and DF node based on the conditions of the channels of source node, relay node, and destination node. It is also shown that the BER and system outage probability of IDAF is better than the HDAF. In paper [20] authors proposed a method to evaluate outage performance of a mixed AF and DF relaying technique in cognitive radio system. Unlike the AF and DF relay technique, the proposed protocol has other or secondary source that tries to transmit its signal to secondary destination with the aid of two secondary relays nodes. Out of the two secondary relays, one relay operates with AF mode and other node operates with DF mode. Results showed that the proposed protocol has the outage performance in between that of the conventional DF and AF. Paper [21] gave us the idea about energy efficient antenna selection in MIMO-OFDM wireless system. To achieve a better performance in terms of energy, a method was proposed by the author named adaptive antenna selection method where active radio-frequency chains and the antenna indexes are selected on the basis of channel condition.

Therefore, in order to meet the growing future wireless communication systems demand, we have explored MIMO-OFDM relay network in practical fading environment. In this paper, we present the impact of the relay network on the performance of MIMO-OFDM systems in terms of spectral efficiency and BER in Rayleigh channel. Rest of the paper is organized as follows. In Section II, proposed system model is described. Analysis 
of performance in terms of capacity and BER of MIMO-OFDM system with relay network is presented in Section III, and Section IV provides the analytical and simulation results. Finally, Section V concludes our work.

\section{SYSTEM MODEL}

The proposed MIMO-OFDM system with relay nodes is shown in Fig.1. We have considered $N$ OFDM modulators and OFDM demodulators with $r$ number of relay nodes. The relay nodes are represented by $R_{l}$, $R_{2}, \ldots, R_{r}$. and the number of relay nodes are assumed to be less than or equal to the number of OFDM modulator and demodulator i.e., $r \leq N$. The relaying techniques which are already discussed in the previous section are implemented on these relay nodes. Moreover, these relay nodes are able to perform decoding or amplification or both with respect to decode and forward, amplify and forward or hybrid decode and forward relaying techniques.

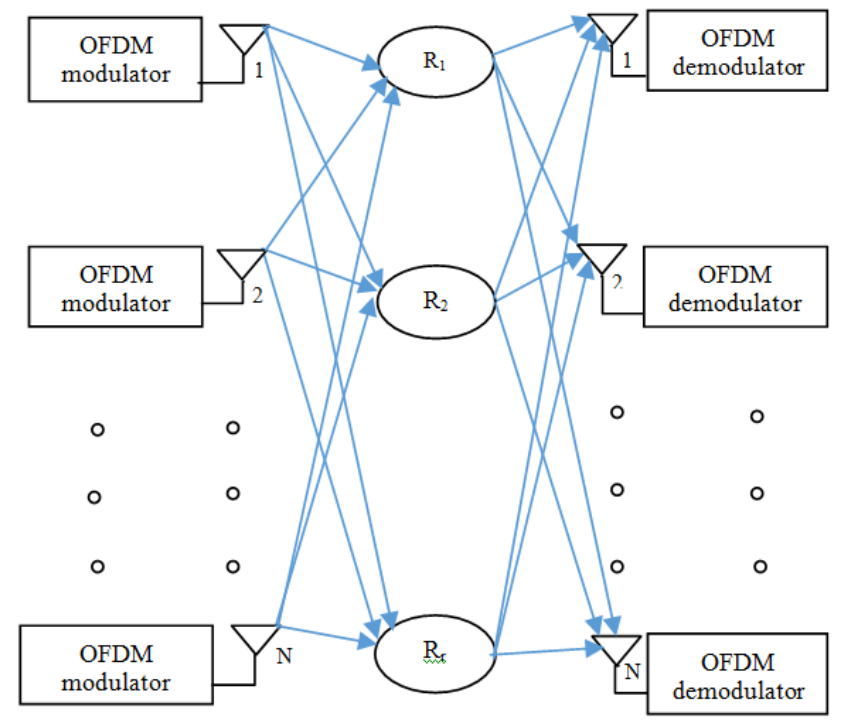

Figure 1. MIMO-OFDM system model with relay nodes

Also some error correcting and detecting techniques like forward error control (FEC) or cyclic redundancy check (CRC) could be applied on the data by these nodes to ensure an error free transmission depending upon the relay protocol used. This MIMO-OFDM system with relay nodes has gained such popularity that the authors in [22] have proposed UV communication with MIMO-OFDM while implementing AF and DF relay nodes. This UV communication focussed on NLOS wireless communication and is mainly considered to abolish tracking and pointing requirements of infrared links and optimal power allocation schemes to increase the performance of the system is presented.

\section{III.ANALYSIS}

In this section, the analysis of proposed system model i.e. MIMO-OFDM system with relay nodes in between the source and destination is presented. We have considered three relaying techniques which are named as amplify and forward, decode and forward, and hybrid relaying. The procedure for simulating the results has been described below for different relaying techniques.

In amplify and forward relay technique, the relay node simply amplifies the data and forwards it to the destination. We have considered data of $n$ bits which is to be transmitted from the source node. At the relay node, cyclic redundancy check (CRC) is performed to detect any kind of error occurring in the system. CRC is best suited for the burst error detection which makes it the optimum choice for the relay. Another feature of CRC is that it broadcasts the data from a single source to multiple destinations which is again well suited for relaying networks. Pilot bits are further added in the data which are known to receiver and sender and helps in reception of signals and decoding process.

However in decode and forward relay technique, again data of $n$ bits is considered. After CRC error detection technique, the data is applied to the Reed Solomon encoder [23] which is used to remove the burst errors. Moreover, the system performance of AF is limited by the noise which is amplified at the relay node during the forwarding process, especially at less SNR values. However, the performance of DF degrades when the relay node fails to interpret the received data signals correctly and this process of decoding and then reencoding causes significant error propagation. In this context, further we have considered the hybrid relaying technique known as hybrid decode amplify and forward (HDAF) which combines the merits of both AF and DF by forwarding the error free and amplified version of the data to the destination node [24]. 
Further, since modulation is necessary for any wireless communication system, we have used three different modulation schemes, BPSK, 16- QAM and 64-QAM for all the three different relaying techniques and have compared there results. Spectral efficiency and BER of all these six combination are presented.

Further, since OFDM system is combined in MIMO therefore the block diagram of OFDM system is shown in Fig. 2. At the OFDM transmitter, first the serial input data is converted to the parallel form and modulated accordingly. Data being in frequency domain after modulation is converted to time domain signal by Inverse Fast Fourier Transform (IFFT) process and parallel to serial conversion of the data is done for further modifications. In addition, cyclic prefix is appended to the data in order to combat (inter symbol interference) ISI [25]. Thereafter, data is transmitted over the channel (for the given system Rayleigh channel is considered) and received data is processed at the receiver end. At the OFDM receiver end, the reverse function of each block at the transmitter side is performed i.e. removal of cyclic prefix, serial to parallel conversion, demodulation, again parallel to serial conversion and then finally we get transmitted information [26]. We have considered Rayleigh fading channel in which there is no significant and dominant propagation of signal along the line of sight path between the transmitter and receiver side. Rayleigh fading is a model used when the surrounding is occupied with many objects that would scatter the radio signals very much before it arrives at the receiver and is one of the most practical channel model.

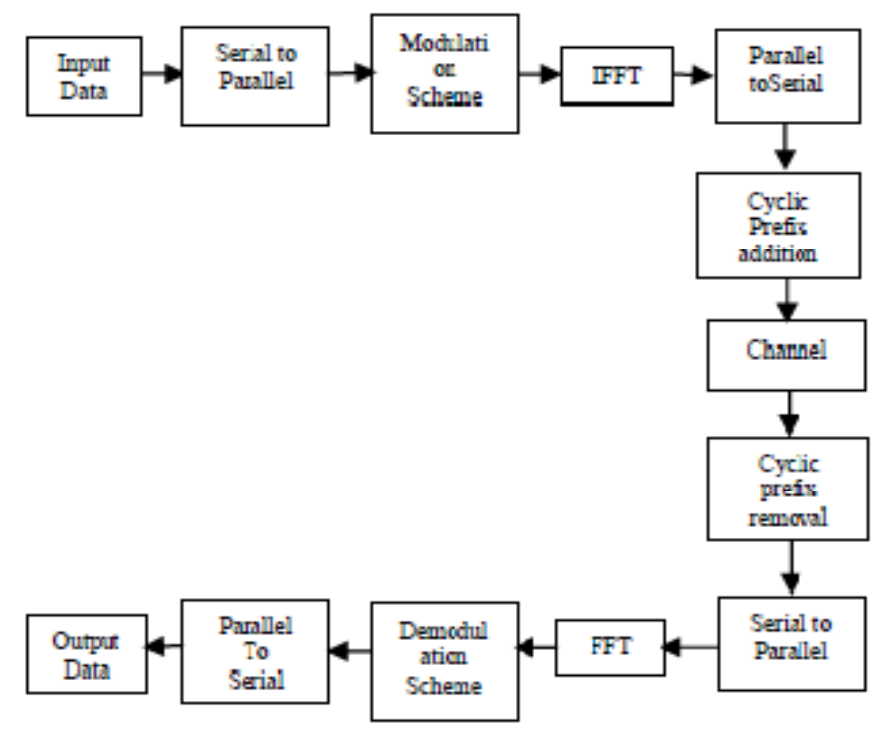

Figure 2. Block diagram of OFDM system

Since we have considered $n$ number of data bits, and let $x(n)$ be the time domain data to be transmitted. Therefore, $X(k)$ is the frequency domain representation of $x(n)$.

At the OFDM transmitter end, IFFT block converts $X(k)$ into $x(n)$ using $x(n)=\frac{1}{N} \sum_{k=0}^{k=N-1} X(k) \cdot e^{j\left(\frac{2 \pi}{N}\right) n k}$ where $N=N$-point IFFT used and $k=0,1, \ldots, N-1$. After the conversion to time domain, addition of guard period i.e. addition of cyclic prefix is done which was explained earlier. The only condition for addition of cyclic prefix is that the length of cyclic prefix should be remarkably more than the delay spread of the channel. If this condition is not satisfied then ISI will have significant effect on the received signal. Further at the receiver end, inversion of the functions of transmitter is performed. For demodulation process, the data is to be converted into frequency domain again. This function is done by the FFT block using $X(k)=\sum_{n=0}^{n=N-1} x(n) \cdot e^{-j\left(\frac{2 \pi}{N}\right) n k}$ where $k=0,1, \ldots N-1$. This explains the OFDM communication system and in order to implement MIMO with this OFDM system, multiple antennas will be employed at both OFDM transmitter and receiver block.

The computation of BER and spectral efficiency for various modulation schemes with three different relaying techniques is carried out. Let $P_{b}$ denotes the error probability, $E_{b}$ is the signal power, $N_{0}$ be the noise power and $\gamma_{b}$ is the SNR per bit. Therefore,

$$
\gamma_{b}=\frac{E_{b}}{N_{o}}
$$

Then BER for BPSK modulation is given as

$$
P_{b}=\frac{1}{2} \operatorname{erfc}\left(\sqrt{\frac{E_{b}}{N_{o}}}\right)
$$


And BER for $M$ - QAM modulations given as

$$
P_{b}=\frac{2(M-1)}{M \log _{2} M} Q\left(\sqrt{\frac{6 \gamma_{b} \log _{2} M}{M^{2}-1}}\right)
$$

where $\operatorname{erfc}($.$) function is related to complementary Gaussian function by following equation:$

$$
Q(x)=\frac{1}{2} \operatorname{erfc}\left(\sqrt{\frac{x}{\sqrt{2}}}\right)
$$

and Spectral Efficiency is given as

$$
C=\log _{2}(1+S N R)
$$

\section{IV.RESULTS AND DISCUSSION}

The simulation parameters which we have considered for our proposed system are: $n$ (number of data bits) =96, $N$ ( $N$-point IFFT or FFT points) =16, $M$ (modulation points) $=16$ and 64. For amplify and forward relaying scheme, 96 data bits are taken and convolution coding is performed. In order to combat ISI in this system, 8 pilot bits are employed. For decode and forward, Reed-Solomon encoding is performed and again 8 pilot bits are considered. However, for HDAF, first decoding then amplification is performed.

We have considered three different modulation schemes namely BPSK, 16-QAM and 64-QAM and have calculated BER and spectral efficiency of the system with these three different modulation schemes. Since spectral efficiency refers to the information rate that can be transmitted over a given bandwidth, therefore 64QAM should have higher spectral efficiency than 16-QAM which in turn will have greater efficiency when compared to BPSK which is shown in the simulation results presented in Fig. 3(a), 4(a) and 5(a). Also BER is computed for these three systems, which is least for BPSK as compared to 16-QAM, which in turn will have less error rate as compared to 64-QAM.

Spectral efficiency and BER of BPSK, 16-QAM and 64-QAM have been simulated using MATLAB software. For BPSK modulation, spectral efficiency and BER are simulated and the simulated results are shown in Fig. 3. It is evident from Fig. 3(a) that hybrid relaying technique used with MIMO-OFDM has greater spectral efficiency in comparison to AF and DF. Also, Fig. 3(b) depicts that hybrid relaying technique has low BER as compared to other relaying techniques.

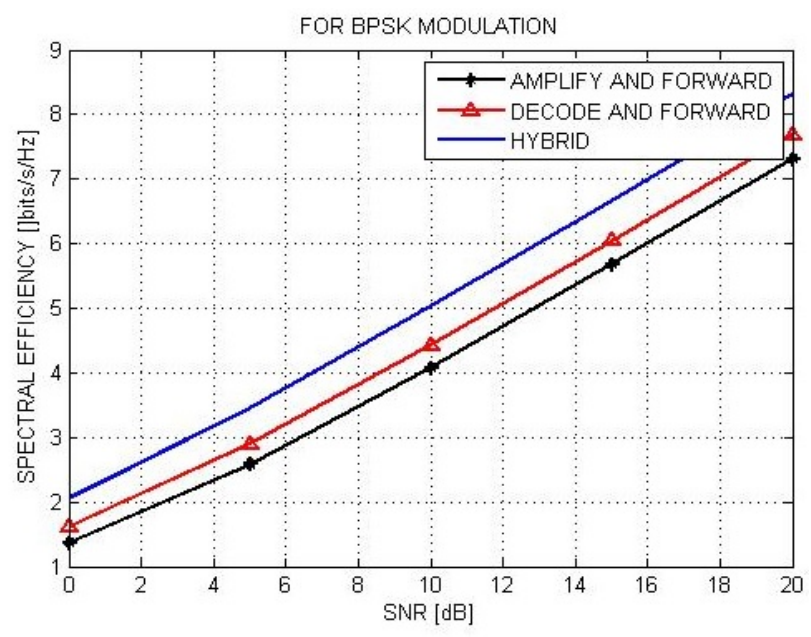

(a) 


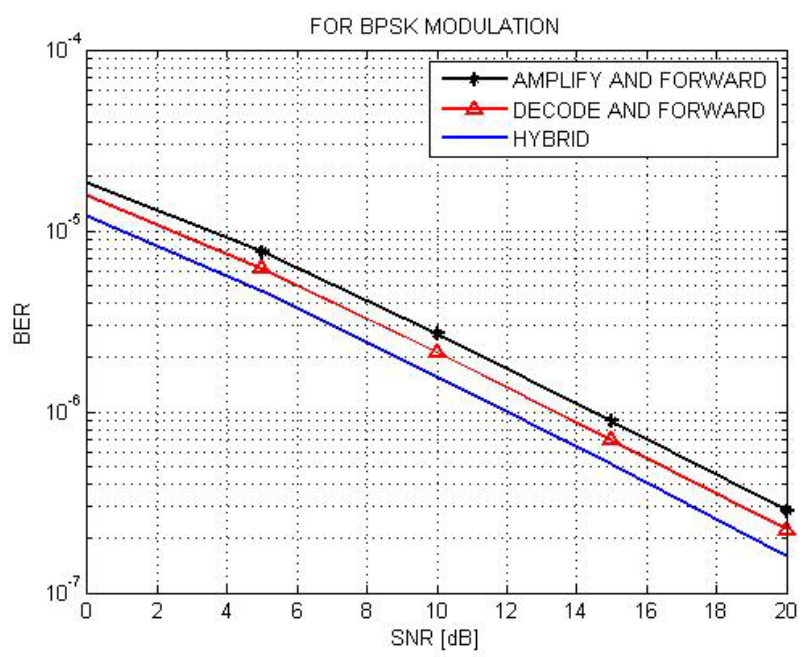

(b)

Figure 3. MIMO-OFDM wireless communication system employing BPSK modulation with AF, DF and HDAF relaying techniques (a) Spectral efficiency versus SNR (b) BER versus SNR.

For 16-QAM modulation MIMO-OFDM relay system, spectral efficiency and BER simulation results are shown in Fig. 4. It is evident from Fig. 4(a) that hybrid relaying technique used with MIMO-OFDM has greater spectral efficiency in comparison to other two relaying methods. Also, Fig. 4(b) depicts that hybrid relaying technique has low BER as compared to other relaying techniques. Further compared to BPSK, the 16-QAM modulation shows higher spectral efficiency, however increase the BER.

Further, for 64-QAM modulation, the similar behavior has been observed in Fig. 5. Further, compared to BPSK and 16 QAM modulation, the 64 QAM shows better performance in terms of spectral efficiency and poor performance in terms of BER.

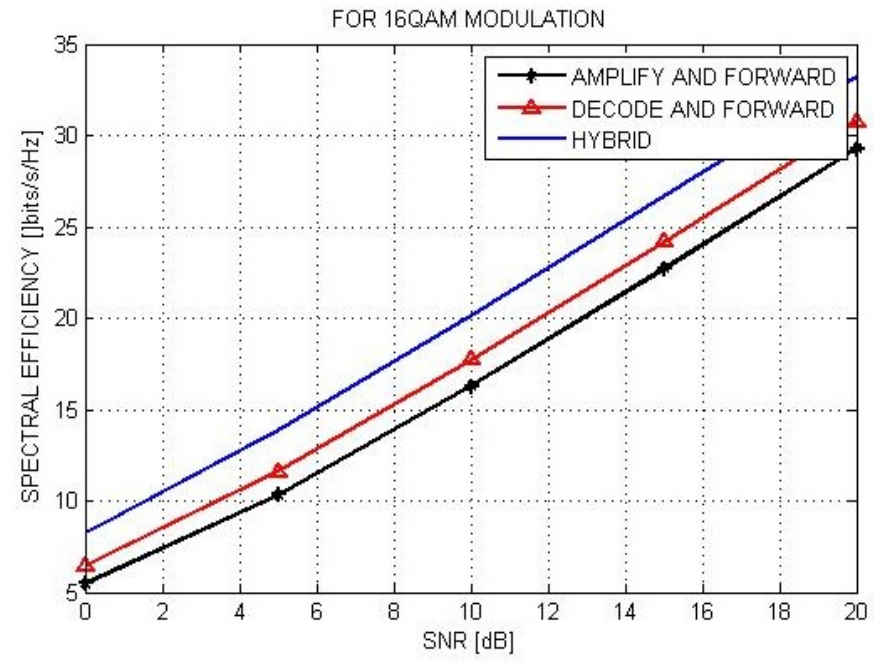

(a) 


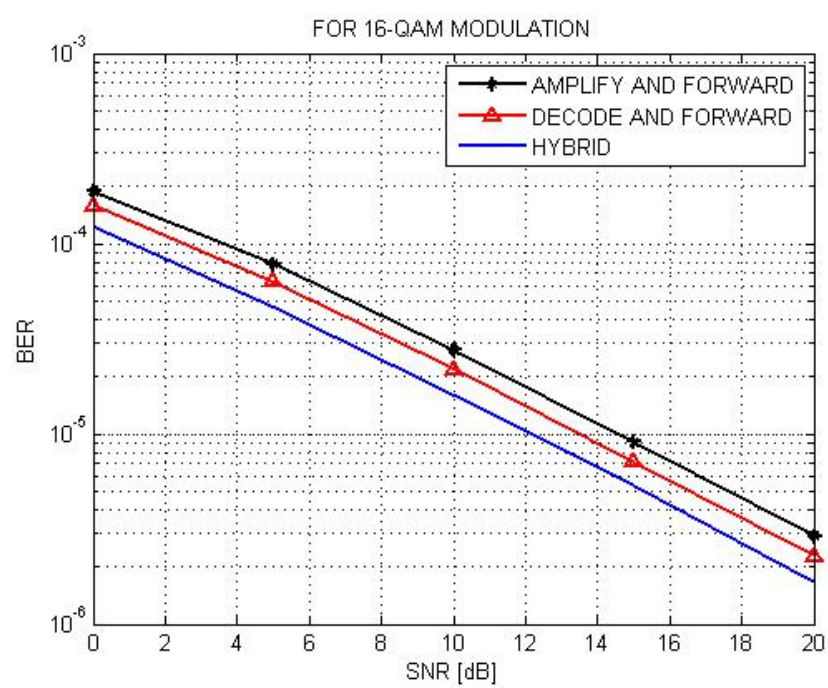

(b)

Figure 4. MIMO-OFDM wireless communication system employing 16-QAM modulation with AF, DF and HDAF relaying techniques a) Spectral efficiency versus SNR b) BER versus SNR.

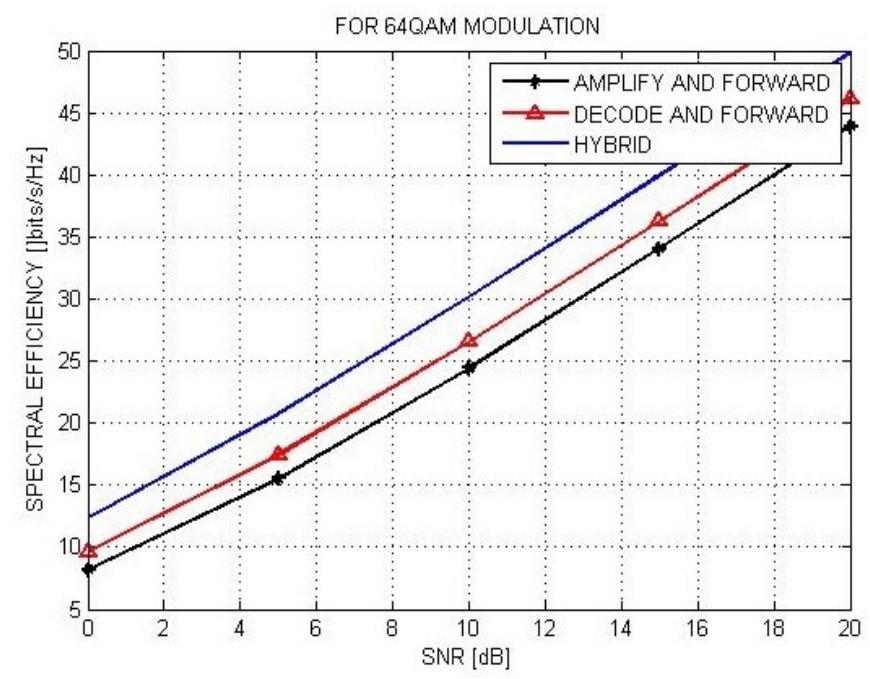

(a)

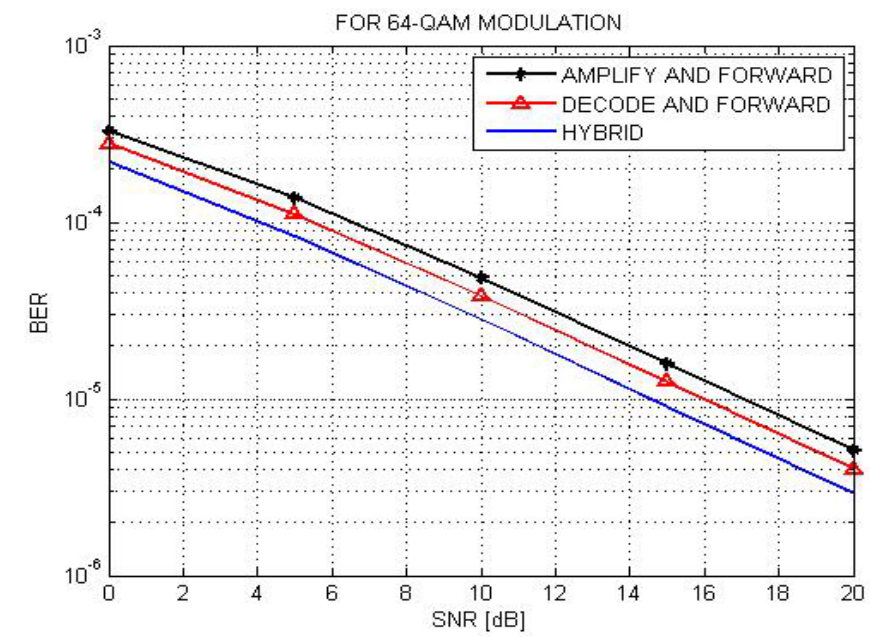

(b)

Figure 5. MIMO-OFDM wireless communication system employing 64-QAM modulation with AF, DF and HDAF relaying techniques a) Spectral efficiency versus SNR b) BER versus SNR. 
Theoretically, HDAF provides more spectral efficiency and less BER in comparison to AF and DF since it combines the advantages of two relaying techniques. Also, since it performs error detection and correction of the data efficiently. Further, it is known that more the modulation order more is the BER and more is the spectral efficiency. Hence, high error probability is the price which has to pay for getting high spectral efficiency.

From the above simulation results it has been concluded that HDAF gives better error rate and spectral efficiency performance in comparison to AF and DF relaying technique. However, comparing different modulation techniques, 64-QAM modulation with HDAF relaying technique gives better spectral efficiency but poor BER than other modulation techniques (i.e. BPSK and 16-QAM) with AF and DF relaying.

\section{CONCLUSION}

In order to improve the BER and the spectral efficiency of the communication system, we have collaborated the MIMO-OFDM system with different relaying techniques. Amplify and forward, decode and forward and hybrid relaying techniques have been considered in our proposed system model. In addition, we have compared the performance of our proposed system under different modulation techniques like BPSK, 16 -QAM and 64QAM. It is concluded from the simulation results that the BER performance is better in BPSK modulated hybrid relaying technique in comparison to 16-QAM and 64-QAM modulation. However, spectral efficiency is better in 64- QAM hybrid relaying technique in comparison to other. Moreover, system complexity and delay are some problems with HDAF relay protocol which we will try to improve in our future communication.

\section{REFERENCES}

[1] C. X. Wang, X. Hong, X. Ge, X. Cheng, G. Zhang, and J. Thompson, “Cooperative MIMO channel models: a survey”, IEEE Communications Magazine, vol. 48, no. 2, pp. 80-87, 2010.

[2] Amit Kumar Sahu*, Sudhansu Sekhar Singh, "BER performance improvement using MIMO technique over rayleigh wireless channel with different equalizers”, Int. J. of Engg. and Tech, vol. 4, no. 5, pp. 333-340, 2012.

[3] K.Rajeswari \#1, M.Anitha \#2, S.J.Thiruvengadam "Channel estimation in MIMO ad hoc network with binomial node distribution and transmit antenna selection”, Int. J. of Engg. and Tech, vol. 6, no. 4, pp. 1811-1817, 2014.

[4] E.Biglier, R.Calderbank, A.Constantinides, A.Goldsmith A.Paulraj, H.Y.Poor, "MIMO Wireless Communications", Cambridge publication, 2007.

[5] A.J.Paulraj, D.A. Gore, R.U. Nabarh, Bolcskei, “An overview of MIMO communications - a key to gigabit wireless”, Proceedings of the IEEE, vol. 92, no. 2, pp. $198-218,2004$.

[6] C.G. Kang, J.Kim, W.Yang, Y.S.Cho "MIMO-OFDM Wireless Communication with MATLAB”, John wiley \& sons, 2010.

[7] H.Sampath, S.Talwar, J.Tellado, V.Erceg, A.Paulraj, "A fourth-generation MIMO-OFDM broadband wireless system: design, performance, and field trial results”, IEEE Communications Magazine, vol. 40, no. 90, pp. 143 - 149, 2002.

[8] Kavitha K V N, A. Jaiswal, S. Khara, "Performance analysis of MISO-OFDM \& MIMO-OFDM Systems", Int. J. of Engg. and Tech, vol. 5, no. 3, pp. 2227-2233, 2013.

[9] K.Song, B.Ji, Y.Huang, Mi.Xiao, L.Yang, "Performance analysis of antenna selection in two-way relay networks", IEEE Transactions on Signal Processing, vol. 63, no. 10, pp. 2520-2532, 2015.

[10] Padmavathi, “Combinational relays with MIMO OFDM using multiple combinational relays”, International Journal of Scientific Research and Education, vol. 3, Issue 3, pp.3109-3117, 2015.

[11] Y. Zhao, R. Adve, and T. J. Lim, "Symbol error rate of selection amplify-and-forward relay systems", IEEE Communications Letters, vol. 10, no. 11, pp. 757-759, 2006.

[12] X. l. Jin, J. No, and D. J. Shin, "Source transmit antenna selection for MIMO decode-and-forward relay networks", IEEE Transactions on Signal Processing, vol. 61, no. 7, pp. 1657-1662, 2013.

[13] T. Q. Duong, H. J. Zepernick, "Hybrid decode-amplify-forward cooperative communications with multiple relays", Proc. of IEEE Wireless Communications and Networking Conference, pp. 1-6, 2009.

[14] K. S. Gomadam, S.A. Jafar, "Optimal relay functionality for SNR maximization in memoryless relay networks", IEEE Journal on Selected Areas in Communication, vol. 25, pp. 390-401, 2007.

[15] R.Titus, U. M. Premkumar, “A survey on incremental relaying protocols in cooperative communication”, International Journal of Research in Engineering and Technology, vol. 03, no.15, pp. 23-30, 2014.

[16] S. S. Ikki and M. H. Ahmed, "Performance of multiple relay cooperative diversity systems with best relay selection over Rayleigh fading channels", EURASIP Journal on Advances in Signal Processing, pp. 1-7, 2008.

[17] L.Fan, X.Lei, T.Q.Duong, M.Elkashlan, G.K. Karagiannidis, "Secure multiuser communications in multiple amplify-and-forward relay networks”, IEEE Transactions on Communications, vol. 62, no. 9, pp 3299-3310, 2014.

[18] X.Jin, J.S.No, D.J Shin, "Source transmit antenna selection for MIMO decode-and-forward relay networks", IEEE Transactions on Signal Processing, vol. 61, no. 7, pp. 1657-1662, 2013.

[19] Z.Bai, J.Jia, C.X.Wang, D.Yuan, "Performance analysis of snr-based incremental hybrid decode-amplify-forward cooperative relaying protocol”, IEEE Transactions on Communications, vol. 63, no. 6, pp. 2094-2106, 2015.

[20] T.T.Duy, H.Y.Kong, "Performance analysis of mixed amplify-and-forward and decode-and-forward protocol in underlay cognitive networks”, IEEE China Communications, vol.13, no.3, pp.115 - 126, 2016.

[21] N.P.Le, F.Safaei, L.C.Tran, "Antenna selection strategies for MIMO-OFDM wireless systems: an energy efficiency perspective", IEEE Transactions on Vehicular Technology, vol.65, no. 4, pp.2048 - 2062, 2016.

[22] M.H.Ardakani, M.Uysal, "Relay-assisted OFDM for ultraviolet communications: performance analysis and optimization", IEEE Transactions on Wireless Communications, vol. 16, no.1, pp. 607 - 618, 2017.

[23] S.B. Wicker, V.K. Bhargava, "Reed-Solomon Codes and Their Applications”, Wiley-IEEE press, 1999.

[24] T.Q. Duong, H.J.Zepernick, "On the performance gain of hybrid decode-amplify-forward cooperative communications", EURASIP Journal on Wireless Communications and Networking, vol. 2009, pp. 1-10, 2009.

[25] H. Bolcskei, "MIMO-OFDM wireless systems: basics, perspectives, and challenges", IEEE Wireless Communications, vol.13, no. 4,pp. $31-37,2006$.

[26] Y. S. Cho, J. Kim, W. Y. Yang, C. G. Kang, “MIMO-OFDM Wireless Communications with MATLAB”, IEEE press, John Wiley \& Sons, 2010. 


\section{AUTHOR PROFILE}

Shivi Tikko received Bachelor of Technology in Electronics and Communication Engineering from U.P.T.U. in 2014, and Master of Technology in Electronics and Communication Engineering from Jaypee Univesity of Information Technology, Waknaghat, Solan in 2017. Her research areas include MIMO systems, wireless communications.

Shweta Pandit received Bachelor of Technology (Hons.) in Electronics and Communication Engineering from Himachal Pradesh University (HPU) in 2010, Master of Technology and Ph.D. from Jaypee Univesity of Information Technology, Waknaghat, Solan in 2012 and 2015 respectively. Her area of interests is cognitive radio and wireless communication.

Pradeep Kumar received Bachelor, Masters and Doctoral degrees in Electronics and Comm. Engg. from Institute of Engineering and Technology, R. U. Campus, Thapar Institute of Engineering and Technology (Deemed University), and Jaypee University of Information Tech., Solan, respectively. He was associated with UAM Madrid, Spain as postdoctoral fellow. He is the author of many research papers published in various national/international journals/conferences. At present, he is working as a faculty member in the Discipline of Electrical, Electronic and Computer Engineering, University of Kwazulu-Natal, Durban, South Africa. His research area includes Design and Analysis of Microstrip Antennas, Antenna Arrays, Wireless Communication, Signal and Image Processing. 